\title{
Nitriles, penicillamine, and substituted prolines
}

\author{
D. S. JACKSON
}

From the Department of Medical Biochemistry, University of Manchester

Until very recently most of the attempts at controlling fibrosis have aimed at influencing collagen metabolism by blocking biosynthesis or by reversing already existing fibrosis. The demonstration of a unique sequence of reactions in collagen biosynthesis gave promise of specific agents aimed at inhibiting one or more of these reactions. Furthermore, there is good evidence that it is not the mere accumulation of collagen which affects the functions of the tissues in which fibrosis occurs but the subsequent cross-linking and stabilization which results in a rigid, metabolically inert structure relatively resistant to degradation even by collagenase. Thus inhibiting the formation of cross-links or splitting existing cross-links also seemed to be promising approaches.

\section{Lathyritic agents}

The discovery of lathyrism affecting the connective tissues was fortuitous, and it was some 25 years before the effects of lathyrogens were shown to be due to the failure of newly synthesized collagen to cross-link (Orbison, 1976). The first lathyrogen, isolated from the seeds of Lathyrus odoratus, was $\beta$-aminoproprionitrile (BAPN) and subsequently other analogous compounds were shown to be effective in blocking the formation of both collagen and elastin cross-links (Fig. 1).

As previously discussed in the paper on cell proliferation and collagen biosynthesis (p. 2), the

$\underset{(\beta \text {-amino proprionitrile }}{\mathrm{NH}_{2}} \stackrel{\mathrm{CH}_{2}}{\mathrm{CH}_{2}} \underset{\left.\mathrm{CH}_{2}\right)}{\mathrm{CH}_{2}} \cdot \mathbf{C N}$

$\mathrm{NH}_{2} \cdot \mathrm{CH}_{2} \cdot \mathrm{CN}$

(amino acetonitrile (AAN))

$\mathrm{NU}_{2} \cdot \mathrm{CH}_{2} \cdot \mathrm{CH}_{2} \cdot \mathrm{CH}_{2} \quad \mathrm{SH}$

( $\beta$-mercaptoethylamine (BMEA))

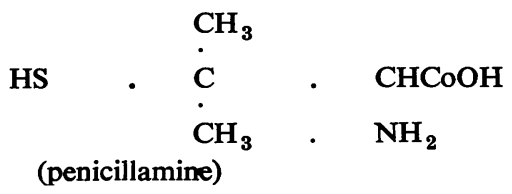

FIG. 1 Lathyritic agents initial reaction in the formation of cross-links involves the enzyme lysyloxidase. The action of this enzyme is inhibited by BAPN. The absence of the aldehyde group on the side chain of lysine or hydroxylysine residues in the collagen polypeptide chain effectively prevents the formation of the cross-links (see A. J. Bailey, 'Some aspects of collagen maturation and fibrosis', p. 5). Thus the physical properties of the fibrous tissue are altered without altering the rate of collagen synthesis.

There have been a large number of successful animal studies in which treatment was simultaneous with the production of fibrosis - that is, while active synthesis was taking place. Only one study (Levene, 1968) showed a substantial reduction of lung fibrosis induced before treatment with BAPN, but this has not been followed up. A few human studieg have been reported with some success in the treate ment of scleroderma with BAPN (Keiser and Sjoerdsma, 1967), the preservation of gliding function in injured tendons (Peacock and Madden, 1969; Bora et al., 1972), and in the prevention of contraction in a lime-burned oesophagus (Davis $e t$ al., 1972; Peacock, 1973). These studies have also shown that BAPN has some toxicity.

\section{Penicillamine}

Penicillamine (Fig. 1), a copper chelator, was introduced as a therapeutic agent for Wilson's disease. A side effect of the therapeutic use of penicillamine was the production of ulcers of the skin over pressure points (Scheinberg, 1964). Subsequent animal experiments indicated that penicillamine was a lathyrogen, the action of which differed from that of BAPN.

Penicillamine acts after the action of lysyloxidase by interacting with aldehyde produced by this enzyme, effectively blocking the next step in the formation of cross-links. It also appeared to break pre-existing cross-links in rats. However, this proved to be fortuitous, since only one type of cross-link is in fact affected and that only before its subsequent stabilization (see paper by A. J. Bailey, p. 5). This failure to act on stabilized cross-links has been underlined by the finding that it is clinically useful in the treatment of scleroderma only in the active phase of the disease when collagen biosynthesis is taking place (Herbert et al., 1974; and see M. I. V. 


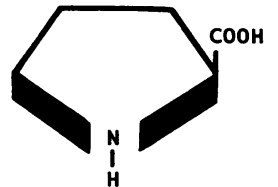

L. PROLINE

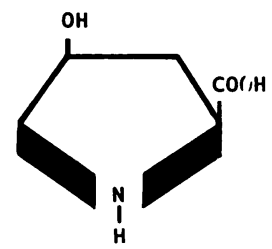

cis - 4 - HYDROXY - L - PROLINE

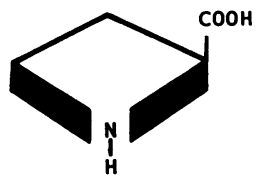

L - AZETIDINE - 2 CARBOXYLIC ACID

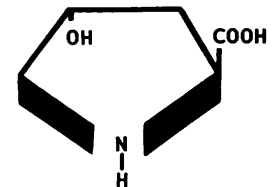

trans - 4 - HYDROXY - L - PROLINE

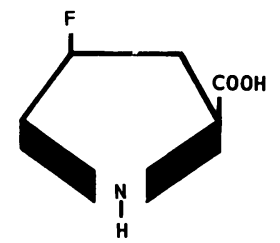

CIS - 4 - FLUORO - HYDROXY L - PROLINE

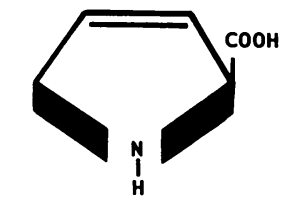

3 - 4 - DEHYDRO - L - PROLINE
FIG. 2 Proline analogues

Jayson, 'Collagen changes in the pathogenesis of systemic sclerosis', p. 26).

Penicillamine has been much used for the treatment of liver fibrosis (Sternlieb, 1973) but despite some reported improvement in hepatic function there has been no evidence of any reduction of fibrosis. This again indicates that once the collagen is laid down and stabilized its cross-links are unlikely to be affected by penicillamine or other agents affecting collagen cross-links.

\section{Proline analogues}

The rationale behind the use of these reagents is that proline transfer RNA cannot distinguish them from the proline and hence they are incorporated into newly synthesized collagen polypeptide chains. Once incorporated, however, they are unable to act as substrates for the enzyme prolylhydroxylase and therefore cannot be hydroxylated. The replacement of hydroxyproline by these analogues reduces the denaturation temperature by some $15^{\circ} \mathrm{C}$. This prevents the formation of the collagen triple helix, prevents the secretion of the abnormal collagen, and renders it readily susceptible to proteolysis (Grant and Jackson, 1976; Prockop et al., 1976; and see D. S. Jackson, 'Cell proliferation and collagen biosynthesis', p. 2).

To date, only animal and cell culture experiments have been reported (Uitto and Prockop, 1975; Chvapil, 1975a), and these have shown that the analogues are incorporated into collagen. However, there are conflicting reports about their effectiveness in inhibiting collagen formation in vivo. Thus Jimenez and Prockop (1971) and others have reported the specific inhibition of collagen synthesis by cis-hydroxyproline (Fig. 2) and Rojkind (1973) the inhibition of liver fibrosis by L-azetidine-2carboxylic acid (Fig. 2).

Others (Madden et al., 1973; Chvapil et al., 1974) were unable to show any specific inhibition of collagen synthesis or accumulation in granuloma, skin wounds, or liver by 3,4 dehydro-hydroxyproline or cis-hydroxyproline (Fig. 2). They reported the production of toxic effects in several biologicalo systems: 3,4 dehydro-hydroxyproline was the more toxic. The reasons for these different results haveo yet to be resolved.

\section{Conclusions}

None of these compounds have been effective in reversing clinical conditions in which fibrosis is already present, although penicillamine may be effective in active scleroderma. BAPN seems to be promising in certain situations where fibrosis can be expected and treatment begun early. Even with BAPN, as with penicillamine, there are problems of toxicity to be overcome. The problem of preexisting chronic fibrosis still remains, and the more fruitful approach here may be the stimulation of collagen catabolism rather than inhibition of synthesis, except perhaps where the collagen concerned is metabolically very active with a high turnover and comparatively short half life. 